\title{
Gamma rays from violent interstellar events
}

\author{
J.B.G.M. Bloemen \\ Leiden Observatory and Laboratory for Space Research Leiden \\ P.O. Box 9513, 2300 RA Leiden, The Netherlands
}

\section{Summary}

As an illustration of what the next generation of $\gamma$-ray telescopes may show us, an up-to-date COS-B 'finding chart' of potential $\gamma$-ray point sources and unexplained extended $\gamma$-ray features is presented. The latter, in particular, may be related to energetic phenomena in the interstellar medium, capable of enhancing the local cosmic-ray density. As an example, a prominent feature, extending over at least $10^{\circ}-15^{\circ}$ almost perpendicular to the Galactic disk, is discussed in some detail, linking it to the giant HII region S54 and Complex C of high-velocity clouds.

\section{Introduction}

Energetic events in the interstellar medium associated with regions of relatively high gas density, such as, supernova-cloud interactions and the collision of high-velocity clouds (HVC's) with the Galactic disk, may be visible as regions of enhanced $\gamma$-ray emission (see, e.g., the review by Morfill and Tenorio-Tagle, 1983). The basic requirement is that the energy supplied is converted into the acceleration of particles with a reasonable efficiency; the interstellar matter in the region of enhanced cosmic-ray (CR) density converts part of the CR energy into $\gamma$ rays. This occurs through collisional nuclear excitation (leading to $\gamma$-ray lines at $\mathrm{MeV}$ energies), inelastic nuclear interactions with the subsequent production of $\pi^{\circ}$ mesons (leading to $\pi^{\circ}$-decay $\gamma$-ray emission), and Coulomb scattering of CR electrons on the nuclei and electrons of the interstellar gas (leading to bremsstrahlung $\gamma$-ray emission). Also, $\gamma$ rays may be produced by inverse-Compton scattering of high-energy $C R$ electrons on the interstellar radiation field. The relative importance of the emission processes is a strong function of $\gamma$-ray energy (for details, see e.g. Stecker, 1971), but in first order approximation the continuum $\gamma$-ray flux from a certain volume is a measure of the product of the average CR density and the gas (or photon) density. Hence, regions of high gas densities (such as molecular clouds), regions with strong radiation fields (such as the immediate surroundings of $O B$ associations in their early stages), and regions with high CR densities (such as probably the evironments of energetic events like supernovae, stellar winds, and violent cloud collisions) have high $\boldsymbol{\gamma}$-ray production rates per unit of volume. The latter, 'active' regions of $\boldsymbol{\gamma}$-ray emission are most pronounced when they coincide with the former two.

The SAS-2 and COS-B $\gamma$-ray surveys $\left(E_{\gamma} \gtrsim 50 \mathrm{MeV}\right)$ show that the appearance of the $\gamma$-ray Milky Way is not dominated by such active regions of $\gamma$-ray emission. A fairly homogeneous distribution of cosmic rays throughout the Galaxy can account in 
good detail for the observed $\gamma$-ray intenstity distribution, with most of the structure attributable to variations in the total gas column density (see review by Bloemen, 1989). Detailed modelling of this diffuse emission, however, reveals several features that are not accounted for.

\section{Unexplained $\gamma$-ray excesses in the COS-B data}

The search for unexplained $\gamma$-ray excesses is closely related to the search for $\gamma$-ray point sources. After some early claims based on balloon experiments, firm detection of $\gamma$-ray point sources was first achieved with the SAS-2 satellite, in particular that of the Crab and Vela pulsars and an hitherto puzzling source, now known as 'Geminga'. The COS-B observations show evidence for several additional sources, which in early stages were simply defined as statistically significant peaks in the $\gamma$-ray intensity distribution, with an angular shape consistent with the COS-B point-spread function (see Hermsen, 1983). This lead to the so called 2CG catalogue (Swanenburg et al., 1981), containing 25 $\gamma$-ray sources. Crab and Vela are unambiguously identified by their timing signatures, but all other identifications have to be based solely on positional coincidence. Given the limited angular resolution (above $100 \mathrm{MeV} \sim 2.5^{\circ} \mathrm{FWHM}$ ) it is difficult to differentiate between intrinsically compact objects and objects that might be up to a few degrees in size, such as CR-irradiated clouds. With the availability of CO surveys (in addition to already existing $\mathrm{HI} 21 \mathrm{~cm}$ data) it became feasible to take this diffuse emission into account in the search for sources, which were then defined as excesses, consistent with the COS-B point-spread function, superimposed on a model of the diffuse emission based on HI and CO surveys (Pollock et al., 1985ab; Simpson and Mayer-Hasselwander, 1987, 1989). Hence, those original sources that find an explanation in terms of the same CRmatter interactions as the underlying diffuse emission (about $30 \%-50 \%$ ) are excluded. Remaining sources may either be intrinsically compact objects or still be of diffuse origin, indicating a high $\mathrm{CR}$ density (our point of interest here) or an enhanced radiation field somewhere along the line of sight.

Evidently, the detection of extended $\gamma$-ray excesses, not particularly aimed at in the analyses of Pollock et al. and Simpson and Mayer-Hasselwander, is of interest for our purposes as well. Also, it is advantageous to combine the $\gamma$-ray data for all energies, which was not done by these authors. The energy range $100 \mathrm{MeV}-5 \mathrm{GeV}$ is a good compromise between counting statistics and angular resolution (which degrades with decreasing energy). Further, allowing more flexibilty in the predicted diffuse emission seems appropriate, because the $\mathrm{CR}$ distribution adopted for the modelling (a function of Galactocentric radius only) is probably a gross approximation. The following describes a first attempt to take these points into account in a simple manner.

Fig. 1 presents a 'finding chart' of potential $\gamma$-ray sources and extended features for the $100 \mathrm{MeV}-5 \mathrm{GeV}$ range. Basically, this map represents the $\gamma$-ray excesses that are left when the expected diffuse emission (from Strong et al., 1988) is subtracted from the observed $\gamma$-ray intensity distribution. It is not a pure subtraction. In order to correct for deviations between model and observations on scales of $10^{\circ}-20^{\circ}$ in longitude direction, the modelled intensities were first re-adjusted to the observed intensities on this scale: for a given position, the predictions in a strip of 21 pixels in longitude and 1 pixel in latitude (pixel size is $0.5^{\circ} \times 0.5^{\circ}$ ), centered on this position, were scaled upwards or 

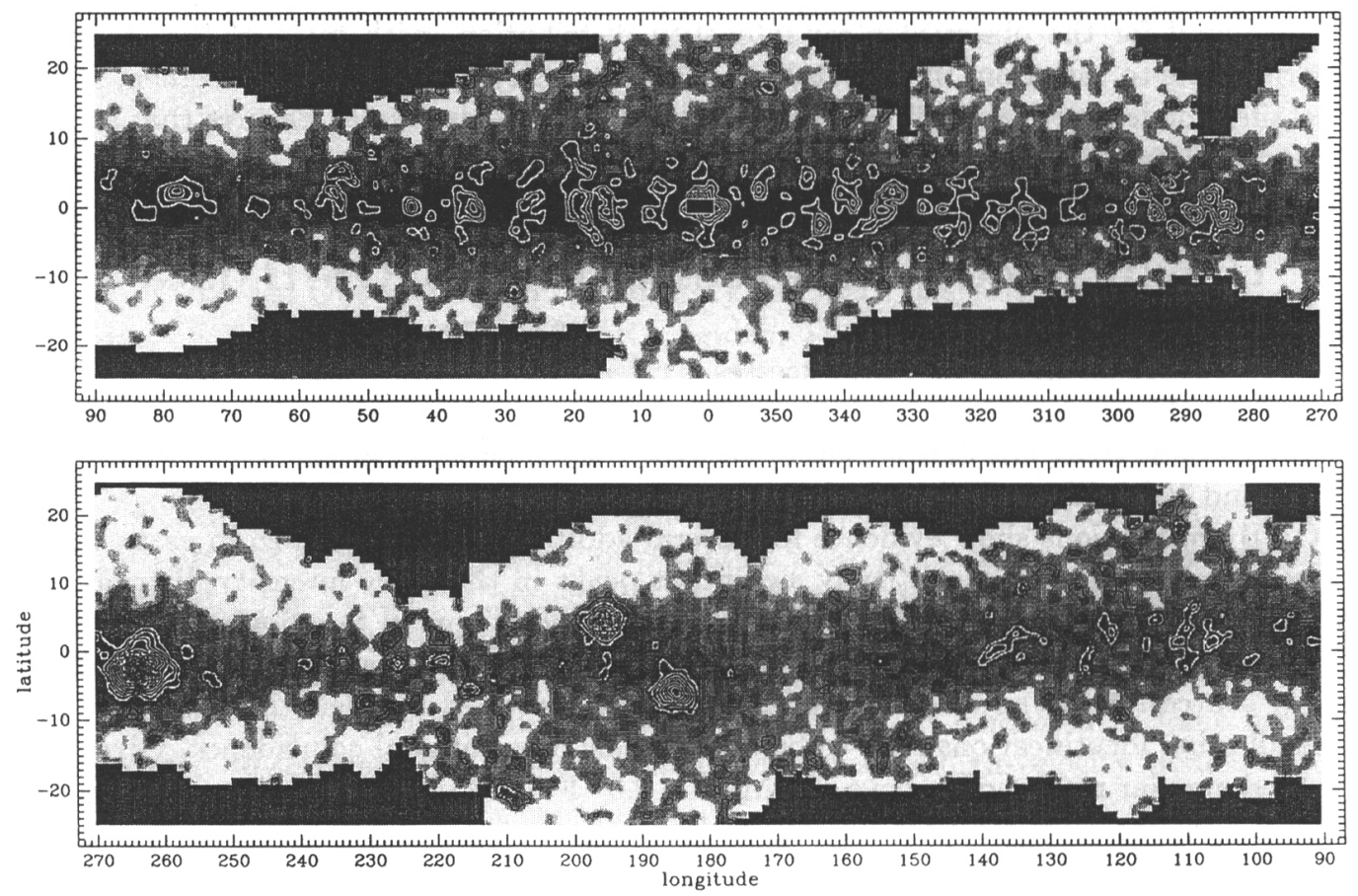

Fig. 1. 'Finding chart' of potential $\gamma$-ray point sources and extended excesses, derived from the COS-B $\gamma$-ray survey $(100 \mathrm{MeV}-5 \mathrm{GeV})$. For details, see Section 2. The underlying gray-scale map represents the observed $\gamma$-ray sky. The small box in the Galactic-centre direction indicates a region that was excluded from the analysis; the feature surrounding it is probably an artifact. The strong excesses near $\ell=185^{\circ}$, $195^{\circ}$, and $263^{\circ}$ correspond to the Crab, Geminga, and Vela $\gamma$-ray sources. Note the extended features visible in the map. Contour values: $8,13,18, \ldots \times 10^{-5}$ photon $\mathrm{cm}^{-2} \mathrm{~s}^{-1} \mathrm{sr}^{-1}$.

downwards such that for only $20 \%$ of the pixels the observed intensities are below the predicted intensities. This approach was chosen, instead of a pure normalization over a certain area, because it will reveal all indications for excesses in the data (the value of $20 \%$, however, is a rather arbitrary choice). Clearly, no formal statistical significance can be assigned to the excesses; 'finding chart' is probably the best denomination for the resultant map. To give the reader some indication of the significance, it may be useful to point out, on the basis of detailed studies of some selected regions, that even excesses with only two contours can be considered of real interest. Less attention should be paid to the precise shape of the excesses. Interestingly, however, some features are clearly extended, most pronounced being the ones near $\ell \approx 15^{\circ}-20^{\circ}$ and $\ell \approx 330^{\circ}-340^{\circ}$.

\section{An example: the excess near $\ell \approx 15^{\circ}-20^{\circ}$}

With a flux of $\sim 2 \times 10^{-6}$ photon $\mathrm{cm}^{-2} \mathrm{~s}^{-1}(100 \mathrm{MeV}-5 \mathrm{GeV})$, this excess would be among the most intense sources in the 2CG catalogue, if not extended. It coincides with two unique phenomena: 'Stockert's chimney', a (thermal) radio-continuum spur 


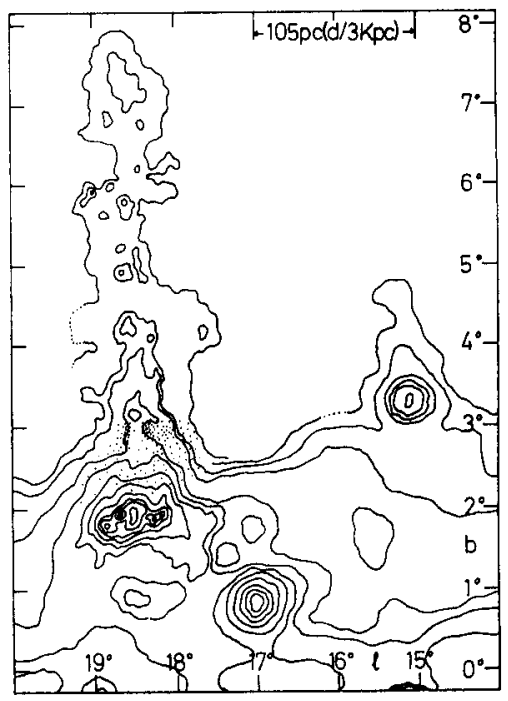

Fig.2. Sketch of 'Stockert's chimney' at $2.7 \mathrm{GHz}$, with $\mathrm{S54}$ located at $\ell \approx 18.5^{\circ}$, $b \approx 2^{\circ}$ (Müller et al., 1987; Kundt and Müller, 1987).

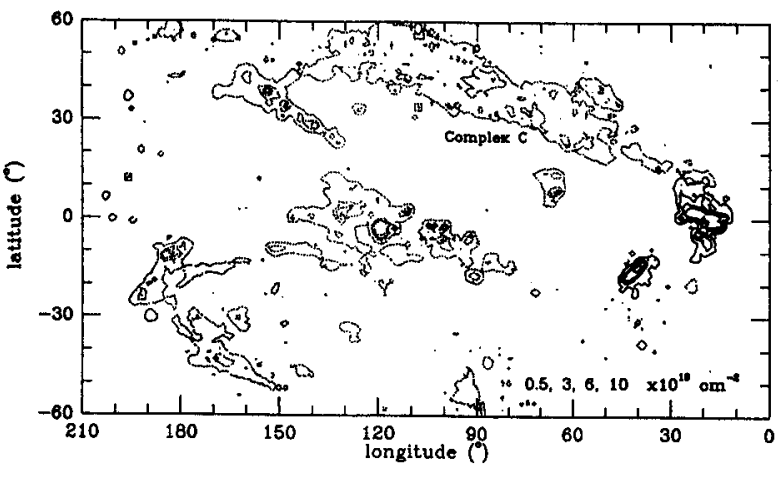

Fig.3. HI column-density map of the HVC's in the catalogue of Hulsbosch and Wakker (1988), illustrating the possible connection between Complex $\mathrm{C}$ and the $\gamma$-ray excess near $\ell \approx 15^{\circ}-20^{\circ}$. Dotted: negative velocities. Full: positive velocities. The so-called Outer Arm complex has been excluded.

associated with the giant HII region S54 (Müller et al., 1987; Kundt and Müller, 1987; see Fig. 2) and the crossing of the extension of the so called Complex C of HVC's with the Galactic disk (Fig. 3). At a kinematic distance of $\sim 3 \mathrm{kpc}$ (the molecular cloud associated with $S 54$ is visible in Fig. 5 at $V \approx 30 \mathrm{~km} / \mathrm{s}$ ), S54 is located at $z \approx 100 \mathrm{pc}$ and the radio spur extends up to $z \approx 400 \mathrm{pc}$. Kundt and Müller argued that the spur is an outflow phenomenon driven by the HII region, consisting of a light relativistic pair plasma (with an admixture of partially ionized hydrogen and cosmic rays), particularly for reasons of energy and absence of recoil and explosively receding fragments. Apart from the energy requirement $\left(E_{k} \approx 10^{54} \mathrm{erg}\right)$, however, these arguments may not apply (i.e., the chimney may consist of 'ordinary' interstellar matter), if the HVC's and the intermediate-velocity gas seen in this region of the sky are to be associated with S54, as suggested by Fig. 4. Shock acceleration of particles in the surroundings of S54 is then certainly plausible, whatever the precise energy source may be. A quite different interpretation of the findings may be the interaction of infalling HVC's with the Galactic disk. It has been suggested that shock ionization, formation of giant HII regions, and shock acceleration accompany such phenomena (e.g. Tenorio-Tagle, 1981; Morfill and Tenorio-Tagle, 1983). At a distance of $3 \mathrm{kpc}$, the CR enhancement (relative to the CR density of $\sim 1 \mathrm{eV} \mathrm{cm}{ }^{-3}$ in the solar vicinity) that is required to explain the excess $\gamma$-ray flux is about $10^{7} / M$, where $M$ (in units of $M_{\odot}$ ) is the gas mass involved. If, for instance, the observed excess flux would originate largely from the HVC's with positive velocities surrounding S54 (Fig. 4c), with a mass of $\sim 5 \times 10^{4} M_{\odot}$ at $3 \mathrm{kpc}$, then the average CR density would be enhanced by more than two orders of magnitude, and the total CR energy in this volume would exceed $10^{53} \mathrm{erg}$. A detailed analysis will be presented elsewhere.

It may be clear that with the launch of the Soviet-French $\gamma$-ray telescope GAMMA-1 and NASA's Gamma Ray Observatory, $\gamma$-ray astronomy can soon be expected to give exciting new insight into energetic interstellar phenomena. 

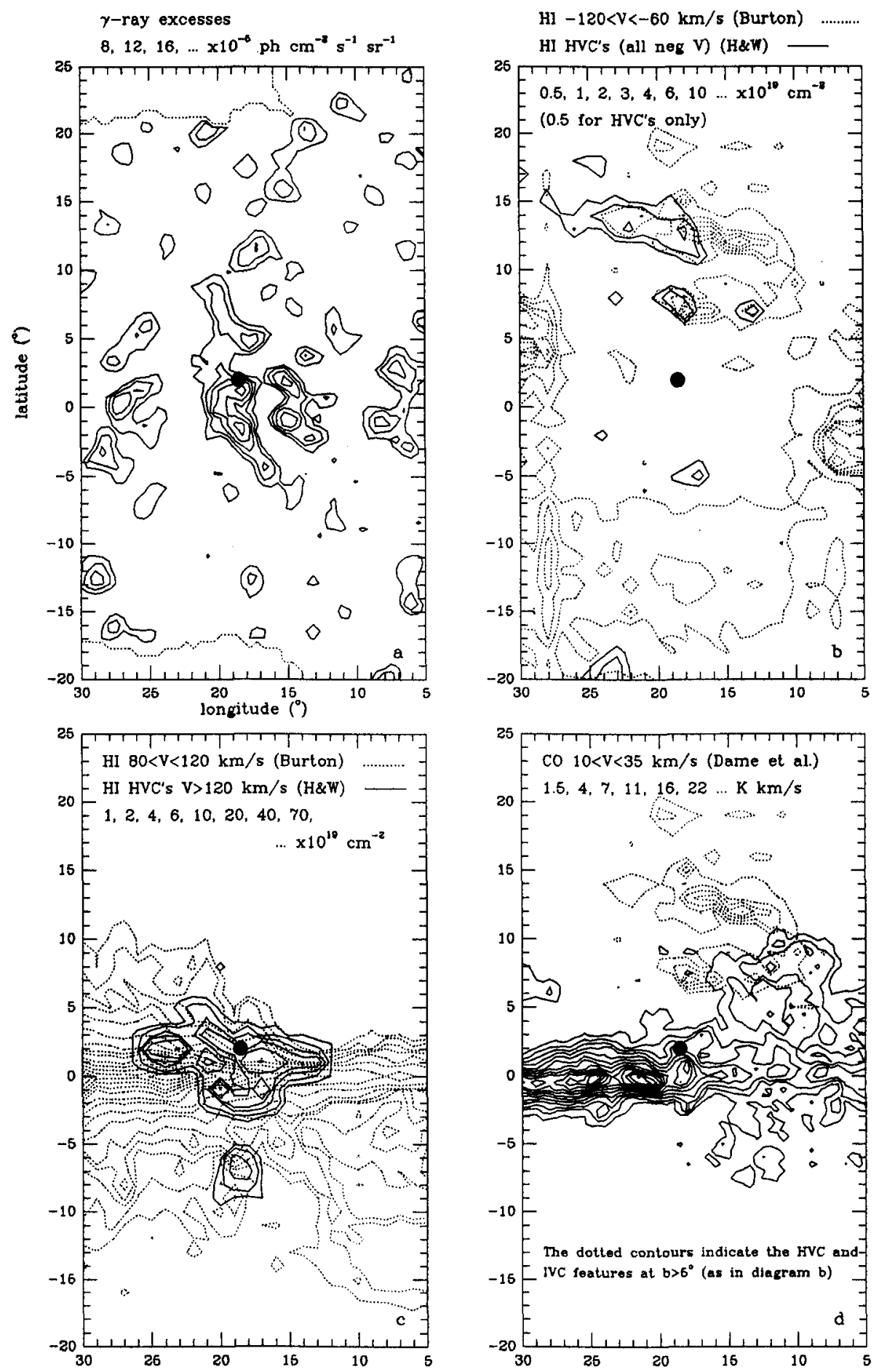

Fig. 4. A compilation of selected $\mathrm{HI}(\mathrm{b}$ and $\mathrm{c}$ ) and $\mathrm{CO}(\mathrm{d})$ observations around the extended $\gamma$-ray excess near $\ell \approx 15^{\circ}-20^{\circ}$ (a). The black dot indicates the position of S54. In Fig. $4 \mathrm{~b}$, the extended lowintensity feature seen at $b<-5^{\circ}$ is probably spurious (see Burton and te Lintel Hekkert, 1985). Also, in Fig. $4 \mathrm{c}$, the feature seen at $b<-10^{\circ}$ (extending to $\ell \approx 350^{\circ}$ ) needs confirmation. 

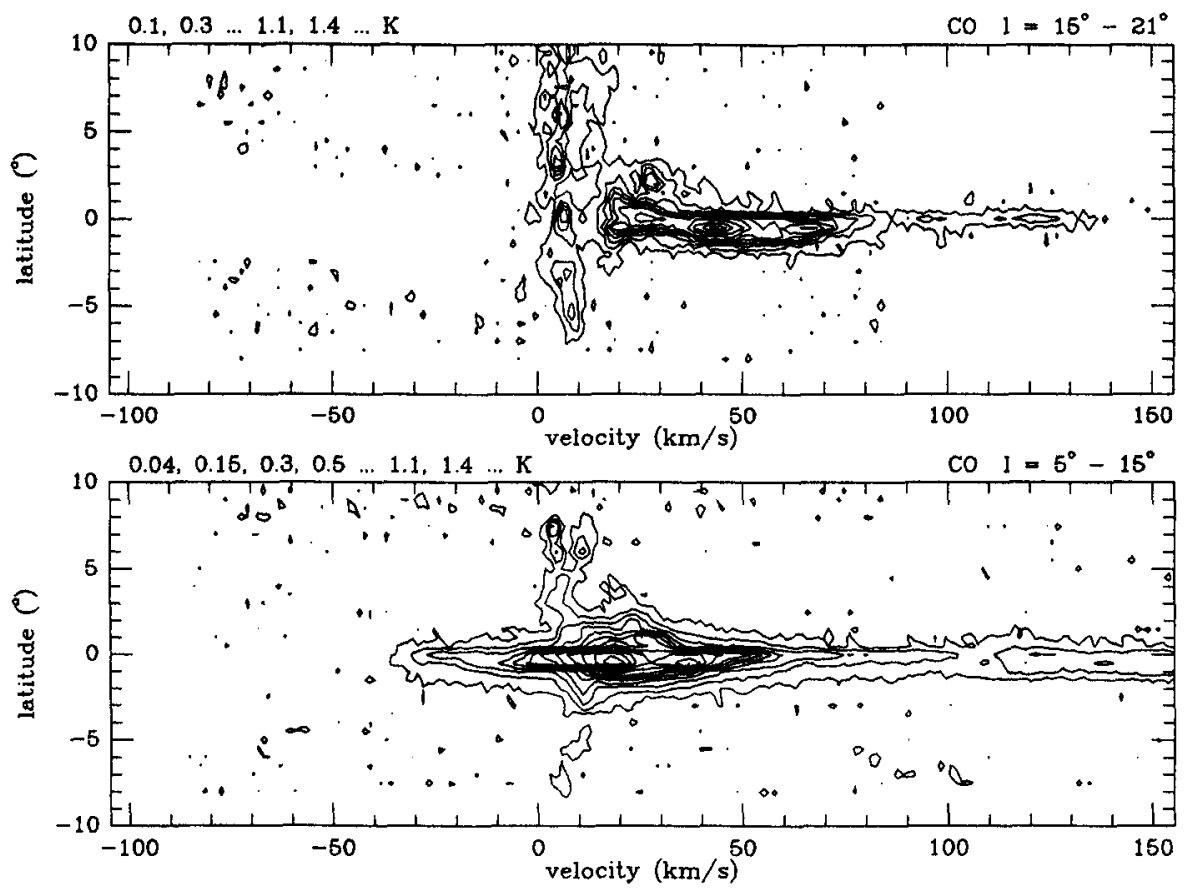

Fig. 5. Latitude, velocity maps of the CO line, averaged over $5^{\circ} \lesssim \ell \lesssim 15^{\circ}$ (lower) and $15^{\circ} \lesssim \ell \lesssim 21^{\circ}$ (upper), derived from the Columbia - Cerro Tololo CO survey (Dame et al., 1987, and references therein). The upper diagram includes the molecular cloud adjacent to $S 54$, visible at $V \approx 30 \mathrm{~km} / \mathrm{s}, b \approx 2^{\circ}$. Note the presence of high-latitude gas at $V>10 \mathrm{~km} / \mathrm{s}$.

Acknowledgements It is a pleasure to thank B.P. Wakker for informative discussions on high-velocity clouds. I acknowledge the receipt of a Fellowship from the Royal Netherlands Academy of Arts and Sciences (KNAW).

\section{References}

Bloemen, J.B.G.M. 1989. Ann. Rev. Astr. Ap. 27 (in press)

Burton, W.B. 1985. Astr. Ap. Suppl. 62:365

Burton, W.B., te Lintel Hekkert, P. 1985. Astr. Ap. Suppl. 62:645

Dame, T.M., Ungerechts, H., Cohen, R.S., de Geus, E. et al. 1987. Ap. J. 322:706

Hermsen, W. 1983. Space Sci. Rev. 36:61

Hulsbosch, A.N.M., Wakker, B.P. 1988. Astr. Ap. Suppl. 75:191

Kundt, W., Müller, P. 1987. Astrophys. Space Sci. 136:281

Mayer-Hasselwander, H.A., Simpson, G. 1989. Adv. in Space Research (in press)

Morfill, G.E., Tenorio-Tagle, G. 1983. Space Sci. Rev. 36:93

Müller, P., Reif, K., Reich, W. 1987. Astr. Ap. 183:327

Stecker, F.W. 1971. 'Cosmic Gamma Rays', Mono Book Corp.

Pollock, A.M.T., Bennett, K., Bignami, G.F., Bloemen, J.B.G.M. et al. 1985. Astr. Ap. $146: 352$

Pollock, A.M.T., Bennett, K., Bignami, G.F. et al. 1985. Proc. 19th Int. Cosmic Ray Conf. 1:338

Simpson, G., Mayer-Hasselwander, H.A. 1987. Proc. 20th Int. Cosmic Ray Conf. 1:89

Strong, A.W., Bloemen, J.B.G.M., Dame, T.M., Grenier, I. et al. 1988. Astr. Ap. 207:1

Swanenburg, B.N., Bennett, K., Bignami, G.F., Buccheri, R. et al. 1981. Ap. J. Lett. 243:L69

Tenorio-Tagle, G. 1981. Astr. Ap. 94:338

van Woerden, H., Schwarz, U.J., Hulsbosch, A.N.M. 1985. in 'The Milky Way Galaxy', eds. H. van Woerden, R.J. Allen, W.B. Burton, Reidel, Dordrecht, p. 387 